\title{
March updates: people and places
}

\section{Göttingen minipigs agreement at Exemplar}

Exemplar Genetics, a commercial provider of biomedical swine models based in Sioux City, Iowa, has announced an agreement with Ellegaard Göttingen Minipigs A/S, a Danish provider of the Gottingen minipig, one of the smallest swine options available to biomedical researchers. The pigs were originally developed at the University of Göttingen in Germany in the 1960s, a cross between the Minnesota minipig, the Vietnamese potbelly pig, and the German landrace pig. In 1992, Ellegaard established the first colony of barrier-bred, microbially defined animals.

Under the agreement, Exemplar will "develop and commercialize genetically modified Gottingen Minipigs as models of human disease on a target-by-target basis," according to a press release.

\author{
Tar Heels to collaborate with Taconic \\ Rensselaer, New York-based mouse \\ model provider Taconic Biosciences \\ has announced a collaboration with the \\ University of North Caroline at Chapel Hill \\ to commercialize animal models developed \\ by UNC researchers. Efforts will be based \\ primarily at the International Center for the \\ Advancement of Translational Science at \\ UNC-Chapel Hill. Initial project began late \\ last year; the initial agreement was set terms \\ for five years.
}

\section{ToxTracker to Charles River \\ Charles River Laboratories has announced an agreement with Toxys to provide North American users with access to the Dutch biotech company's ToxTracker assay, an in vitro genetic toxicology assay which can be used to help determine}

the safety of a chemical in question. According to a press release, ToxTracker has shown $94 \%$ sensitivity and $95 \%$ specificity in making toxicity determinations, through validation with reference compound laboratories recommended by the US EPA's Toxicity Forecaster and the EU Reference Laboratory for alternatives to animal testing (EURL ECVAM).

\section{Thinking about Denver}

The $70^{\text {th }}$ Meeting of the American Association for Laboratory Animal Science will take place this coming October in Denver, Colorado, with a Spotlight Forum on "One HealthConnections Between Animal, Human, and Environmental Health." It may still be a few months away, but the Association is calling for topics, abstracts, and award nominations. Submissions for Topics for the National Meeting are due March 15 and should include a brief summary, target audience, and what the attendees will learn. Abstracts for either poster or platform sessions are due by June 1 .

AALAS is also looking nominations for its annual awards: The Nathan R. Brewer Lifetime Achievement Award; the Charles A. Griffin Award; the Joseph J. Garvey Management Award; the Pravin N. Bhatt Scientific Investigator Award; the George R. Collins Education and Training Award; and the Technician of the Year Award. Nominations are due by April 3.

Visit www.aalas.org for more information and to submit materials.

Published online: 19 February 2019 https://doi.org/10.1038/s41684-019-0247-4 